\title{
卫矛科植物抗肿瘤活性物质蛇藤 酯甲的结构测定（II）
}

刘吉开 吴大则* 贾忠建 周 俊* 朱子清

（兰州大学有机化学研究所; *中国科学院昆明植物研究所）

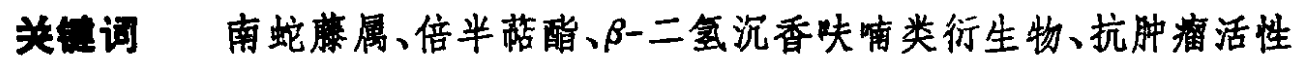

自从卫矛科植物中发现抗癌活性物质一美登素 (maytansine) 和雷公藤甲素 (tripto lide）以来,世界各国科技工作者对从卫矛科植物中寻找新的抗癌活性物质有极大的兴趣. 作 者从粉叶南蛇藤（Celastrus glaucophyllus）根皮的丙酮提取物中,通过溶剂分配处理以及年 化铝和硅胶柱层析分离得到四个倍半萜酯. 应用紫外、红外、高分辨质谱、核磁共振谱和二维 核磁共振谱以及化学方法推定了它们的化学结构, 均是首次发现的新化合物. 本文报道其中 两个倍半萜酯的化学结构. 化合物 1 (蛇藤酯甲, celaglaumin) 经药理篮选发现对 $\mathrm{L}_{1210}$ 和 P388 瘦株具有抑制作用: $1 \mathrm{C}_{50}(\mu \mathrm{g} / \mathrm{ml}), \mathrm{L}_{1210} 2.11 ; \mathrm{P}-3884.12$.

化合物 1 为无色块晶, m. p. $222-223^{\circ} \mathrm{C},[\alpha]_{D}^{20}-48.5^{\circ}\left(\mathrm{C} 0.2, \mathrm{CHCl}_{3}\right)$. 由高分辩质

表 1 蛇藤酧甲的 ${ }^{1} \mathrm{H}$ 和 ${ }^{3} \mathrm{C}-\mathrm{NMR}$ 化学位移数据

\begin{tabular}{|c|c|c|c|c|c|}
\hline 位 1 & $\begin{array}{l}{ }^{13} \text { C-NMR } \\
\text { 化学位移 }\end{array}$ & ${ }^{1} H-N M R$ 化学位移 & 位 置 & $\begin{array}{l}{ }^{13} \mathrm{C}-\mathrm{NMR} \\
\text { 化学位移 }\end{array}$ & ${ }^{\mathrm{I}} \mathrm{H}-\mathrm{N} M \mathrm{R}$ 化学位移 \\
\hline 1 & 71.4 & $5.56 \mathrm{~d}(3.8)$ & 14 & 26.0 & $1.55 \mathrm{~s}$ \\
\hline 2 & 69.5 & $5.65 \mathrm{~m}$ & 15 & 31.4 & 1.568 \\
\hline 3 & 26.6 & $2.36 \mathrm{~m}$ & oco & 169.4 & 1 \\
\hline 4 & 41.7 & $1.75 \mathrm{~m}$ & $\mathrm{CH}_{3}$ & 20.7 & $1.70 \mathrm{~s}$ \\
\hline 5 & 91.6 & & oco & 175.8 & \\
\hline 6 & 76.4 & $5.52 \mathrm{~d}(3.6)$ & $\mathrm{CH}$ & 33.2 & $2.39 \mathrm{~m}$ \\
\hline 7 & 55.2 & $2.49 \mathrm{dd}(3.6,3.0)$ & $\mathrm{CH}$, & 16.7 & $1.13 \mathrm{~d}(7)$ \\
\hline 8 & 77.0 & $4.97 \mathrm{~d}(3.0)$ & $\mathrm{CoH}_{2}$ & 26.6 & \\
\hline 9 & 73.2 & 5.088 & СH, & 11.6 & $0.88 t(7.3)$ \\
\hline 10 & 48.9 & & Ar & $128-133(12 C)$ & $7.3-7.7(6 \mathrm{H}, \mathrm{m})$ \\
\hline 11 & 20.4 & $1.54 \mathrm{~s}$ & co & 164.9 & $8.0-8.1(4 \mathrm{H}, \mathrm{m})$ \\
\hline 12 & 19.1 & $1.41 \mathrm{~d}(8.5)$ & & 164.7 & \\
\hline 13 & 82.3 & & & & \\
\hline
\end{tabular}

相是 $\mathrm{CDCl}_{3}$, 括号内是偶合常数 $(\mathrm{Hz})$, 化学位移为 $\mathrm{ppm}$. 内标 TMS, 仪器是 $\mathrm{AC}-200$ 型.

本文 1988 年4月9日收到. 
谱得其分子式为 $\mathrm{C}_{36} \mathrm{H}_{44} \mathrm{O}_{10}$ (计算值：636.2934, 实测值：636.2867). $\lambda_{\text {max }}\left(\mathrm{CH}_{3} \mathrm{OH}\right) ： 282$ $(\log 83.2), 274(3.25), 232(4.4) \mathrm{nm} . \quad \nu_{\max }(\mathrm{KBr}): 3500$ (br., OH), 1740(br., RCOO-), $1600,1588,1500(\mathrm{~S}, \mathrm{Ar}) \mathrm{cm}^{-1} \cdot m / z: 636\left(\mathrm{M}^{\dagger}\right), 621\left(\left[\mathrm{M}-\mathrm{CH}_{3}\right] \div\right), 515\left(\left[\mathrm{M}-\mathrm{C}_{7} \mathrm{H}_{5} \mathrm{O}_{3}\right] \div\right), 105$

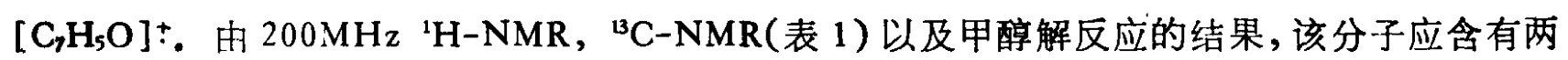
个苯甲酰基, 一一个乙酰基和一个 $\alpha$-甲基丁酰基,其分子母核属于 $\beta$-二氢沉香呋喃类型 ${ }^{[1,2]}$.

在化合物 1 的 'H-NMR 中, H-9 表现为单峰, 在此类化合物中类似这样的情况文献中 也曾报道过 ${ }^{[3]}$. 这可能是环上取代基的影响使环发生一定程度上的扭转从而使 $\mathrm{H}-8$ 和 $\mathrm{H}-9$ 间的双面夹角等于或近于 $90^{\circ}$, 使它们间的偶合常数接近零.

在化合物 1 的 NOESY 中(图 1), 我们观察到 $\mathrm{H}-8$ 和 $\mathrm{H}-7, \mathrm{H}-8$ 和 8-OH, $\mathrm{H}-8$ 和 $\mathrm{OAc}$; H-9 和 OAc 之间有相关峰. H-6 和 H-9, H-6 和 H-7, H-6 和 H-12 之间也有相关峰, 其中 H-6 和 H-9 间之所以会产生相关峰是因为 $9 \alpha-\mathrm{OAc}$ 和 $14-\mathrm{CH}_{3}$ 间空间位阻作用使环 $\mathbf{A}$ 由椅式

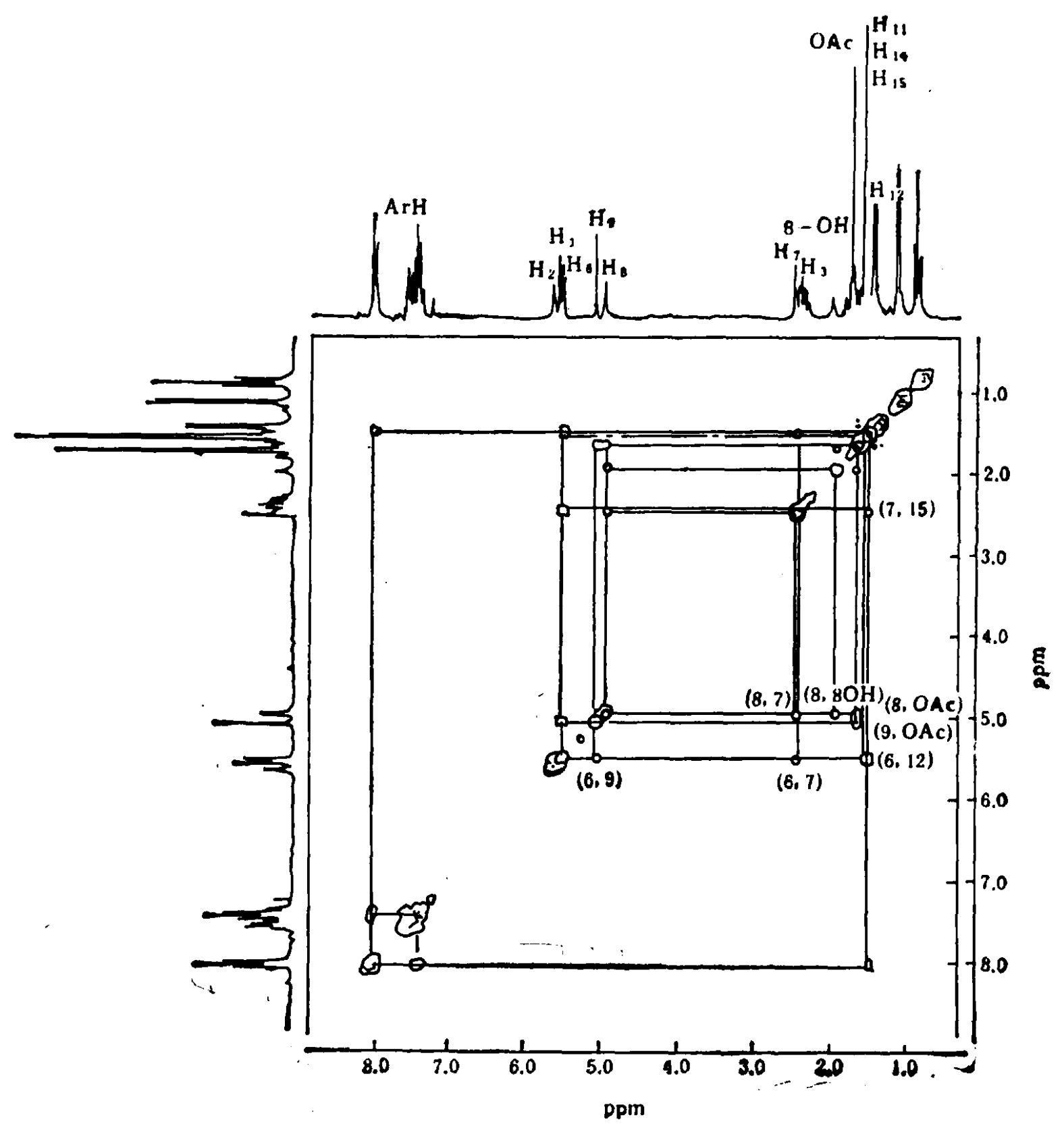

图 1 蛇藤酷甲的 NOESY 
构象转为船式构象, 这样 H-6 和 H-9 间的空间距离接近. 此外, 苯环上的芳空和 H-12 以及 环经之间也有相关峰. 由此推定了该化合物的结构 (1, 图 2)。 ${ }^{1} \mathrm{H}-\mathrm{NMR}$ 和 ${ }^{3} \mathrm{C}-\mathrm{NMR}$. 中各 峰的归属是基于 ${ }^{1} \mathrm{H}-{ }^{-1} \mathrm{H}-\cos Y$ 和 ${ }^{1} \mathrm{H}-{ }^{-1} \mathrm{C}-\mathrm{COSY}$.

将 $50 \mathrm{mg} 1$ 溶于 $0.5 \mathrm{ml}$ 甲醇,加入 $2 \mathrm{ml}$ 新鲜制备的甲醇钠一甲醇溶液，室温下反应，放置过 夜, 反应物用制备薄层纯化得约 $20 \mathrm{mg}$. $90 \mathrm{MHz}^{1} \mathrm{H}-\mathrm{NMR}$ (溶剂 $\mathrm{C}_{5} \mathrm{D}_{5} \mathrm{~N}$, TMS 内标, 8 为 $\mathrm{ppm}$ ): 3.7-5.0(5H, m, $5 \times \mathrm{CHOH}), 2.11,2.03,1.95$ (各 $3 \mathrm{H}, \mathrm{S}, \mathrm{H}-11, \mathrm{H}-14$ 和 $\mathrm{H}-15$ ), $2.01(3 \mathrm{H}, d, J=8 \mathrm{~Hz}, \mathrm{H}-12) . m / z: 302[\mathrm{M}]+, 287\left[\mathrm{M}-\mathrm{CH}_{3}\right] \div, 251\left[\mathrm{M}-\mathrm{CH}_{3}-2 \mathrm{H}_{2} \mathrm{O}\right]+, 233$, $195,145,59,43$.
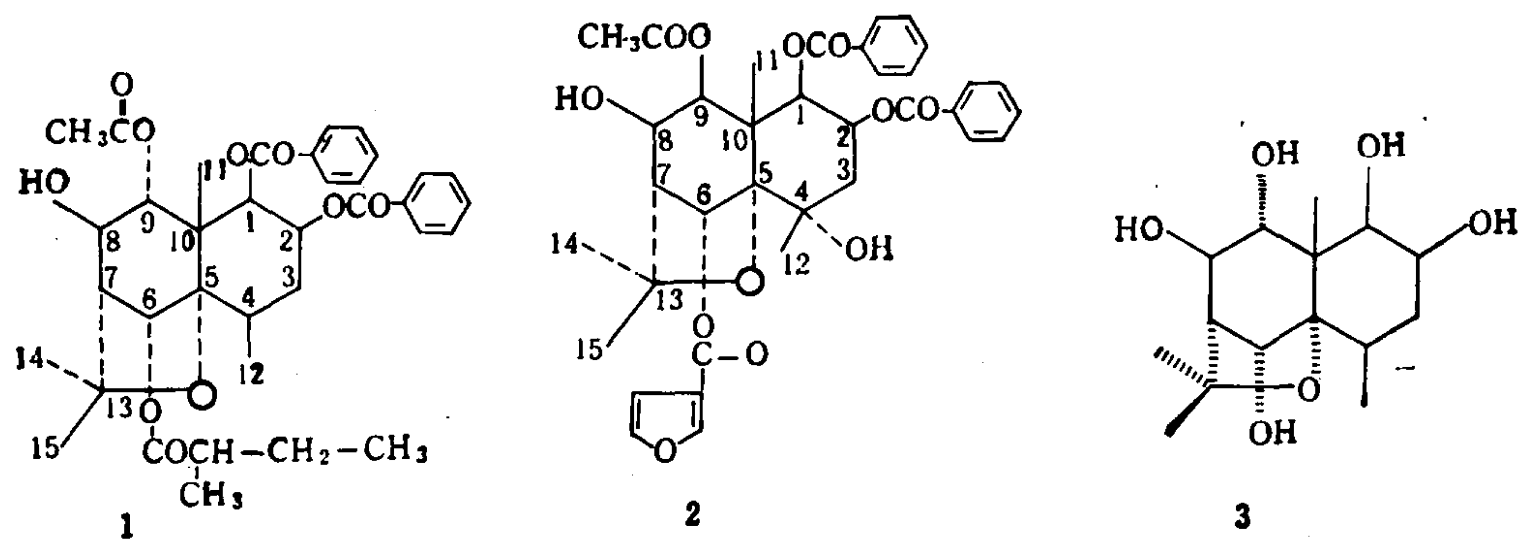

图 2

化合物 2 得量较少 (从 $2 \mathrm{~kg}$ 根皮中得约 $8 \mathrm{mg}$ ), $\lambda_{\text {max }}(\mathrm{EtOH}): 280(\log 83.2), 274(3.3)$, 232(4.5). $v_{\max }(\mathrm{KBr}): 3500(\mathrm{br} ., \mathrm{OH}), 1740$ (br., 酯基), $1600,1588,1500(\mathrm{~S}, \mathrm{Ar}) \mathrm{cm}^{-1} . \mathrm{m} / \mathrm{z}$ : $647\left(\left[\mathrm{M}-\mathrm{CH}_{3}\right]^{+}\right), \quad 629\left(\left[\mathrm{M}-\mathrm{CH}_{3}-\mathrm{H}_{3} \mathrm{O}\right]^{\ddagger}\right) \quad 525\left(\left[\mathrm{M}-\mathrm{CH}_{3}-\mathrm{C}_{7} \mathrm{H}_{6} \mathrm{O}_{2}\right]^{+}\right), \quad 105\left(\left[\mathrm{C}_{7} \mathrm{H}_{5} \mathrm{O}\right]^{+}\right), \quad 95$ $\left(\left[\mathrm{C}_{5} \mathrm{H}_{3} \mathrm{O}_{2}\right]^{+}\right) . \quad{ }^{1} \mathrm{H}-\mathrm{NMR}\left(\mathrm{CDCl}_{3}\right): 5.55(1 \mathrm{H}, d, J=3.7 \mathrm{~Hz}, \mathrm{H}-1), 5.78(1 \mathrm{H}, d d, J=3.7$, $6.5 \mathrm{~Hz}, \mathrm{H}-2), 2.25(2 \mathrm{H}, m, \mathrm{H}-3), 5.58(1 \mathrm{H}, d, J=3.0 \mathrm{~Hz}, \mathrm{H}-6), 2.62(1 \mathrm{H}, d, J=3.0 \mathrm{~Hz}$ $\mathrm{H}-7), 4.95(1 \mathrm{H}, d, J=5.4 \mathrm{~Hz}, \mathrm{H}-8), 5.09(1 \mathrm{H}, d, J=5.4 \mathrm{~Hz}, \mathrm{H}-9), 5.16(1 \mathrm{H}, \mathrm{S}, 8-\mathrm{OH})$ $3.32(1 \mathrm{H}, \mathrm{S}, 4-\mathrm{OH}), 1.90\left(3 \mathrm{H}, \mathrm{S}, \mathrm{CH}_{3} \mathrm{CO}\right), 1.80(3 \mathrm{H}, \mathrm{S}, \mathrm{H}-11), 1.68(3 \mathrm{H}, \mathrm{S}, \mathrm{H}-12), 1.65$ (3H, S, H-14), $1.57(3 \mathrm{H}, \mathrm{S}, \mathrm{H}-15)$. 此外还有呋喃酰基 $(3 \mathrm{H}, 6.7,7.5,7.9)$, 苯甲酰基 $(6 \mathrm{H}, m, 7.3-7.7 ; 4 \mathrm{H}, \mathrm{m}, 8-8.1)$. 化合物 2 的结构和我们报道的另外两个化合物结构十分 近似 ${ }^{(4)}$, 其紫外,红外,核磁共振谱也较类似，它们属于同系列化合物。化合物 2 被命名为蛇藤 酯丁 (Celaglausin).

\section{今 考鳝}

[1] Rzemer, A., Thomas, H. and Budzikiewiez, H., Z. Naturforsch., 31B (1976), 607.

[2] Wagner, H., Heckel, E. and Sonnerbichler, J. Tetrahedron, 31(1975), 1949.

[ 3 ] Baxter, R. L. et al, J. Chem. Soc., Perkin I, 1979, 2972.

[4] Liu Ji-kai, Jia Zhong-jian, Wu Da-gang, Zhou Jun, Zhu Zi-qing and Koenig, W. A.. Z. Naturforsek, 1988 (iv press). 